\title{
Quand compatir tient lieu de faire. Les paradoxes
} sociologiques de la compassion

\section{When compassion is action. The sociological paradoxes of compassion \\ Cuando compadecerse da paso al hacer Las paradojas sociológicas de la compasión}

\section{Marc-Henry Soulet}

Numéro 65, automne 2018

La compassion : souci-pour-l'Autre. Enjeux théoriques et considérations pratiques

URI : https://id.erudit.org/iderudit/1070138ar

DOI : https://doi.org/10.7202/1070138ar

Aller au sommaire du numéro

Éditeur(s)

Athéna éditions

ISSN

0831-1048 (imprimé)

1923-5771 (numérique)

Découvrir la revue

Citer cet article

Soulet, M.-H. (2018). Quand compatir tient lieu de faire. Les paradoxes sociologiques de la compassion. Cahiers de recherche sociologique, (65), 25-46. https://doi.org/10.7202/1070138ar
Résumé de l'article

L'objectif de cette contribution portant sur la compassion est d'examiner ce que cette notion veut dire d'un point de vue sociologique, ce qu'elle nous apprend et permet de comprendre de l'activité sociale. Cet article vise en ce sens à identifier les vertus sociologiques de la compassion, en dégageant ce qu'elle recouvre et ce à quoi elle engage à la lumière de deux variables : d'une part, sa qualité de relation sociale et, d'autre part, son rapport à l'action. Il en ressort une idée-force : la compassion est avant tout une relation sociale, mais a) une relation sociale paradoxale qui égalise sur la base d'une différence maintenue, b) une relation sociale marquée du sceau de l'impuissance en même temps qu'appelant une action et c) une relation momentanée de reconnaissance d'autrui. Ce texte se conclut par l'énoncé de quelques idées explicatives de l'origine de l'impuissance au coeur de la compassion. 


\title{
Quand compatir tient lieu de faire. Les paradoxes sociologiques de la compassion'
}

\author{
MARC-HenRY SOULET
}

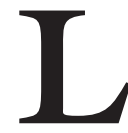

a compassion a pris place dans la vie politique des démocraties contemporaines $^{2}$ avec, notamment, la prégnance de l'humanitaire désormais plébiscité comme modalité alternative d'action devant l'incapacité des États-nations à faire face aux nouveaux défis mondiaux - liés notamment aux transformations climatologiques, aux questions migratoires et aux nouvelles formes de conflits armés - mais aussi vu comme succédané d'une action politique jugée par trop défaillante ou corrompue ${ }^{3}$. Cette présence de la compassion sur la scène sociopolitique doit aussi beaucoup à l'émergence spectaculaire du care et du prendre soin comme philosophie d'un rapport nouveau au monde basé sur la vulnérabilité, faisant de la rela-

1. Cet article reprend et amplifie un argumentaire développé dans une contribution précédente, en apportant notamment un éclairage complémentaire sur l'origine de l'impuissance à agir dans la compassion. Marc-Henry Soulet, «La compassion: un faux ami pour l'analyse sociologique», dans Marina D’Amato (a cura di), Ragioni e sentimenti, Roma, RomaTre Press, 2016.

2. À un tel point que certains auteurs ont pu parler de «déferlement compassionnel» et de «triomphe de la compassion», et voir en la compassion "une valeur essentielle de nos sociétés contemporaines». Myriam Revault d'Allones, «Le zèle compassionnel de Nicolas Sarkozy», Esprit, novembre, 2011, p. 143 et 154; Clifford Orwin, «Le triomphe de la compassion», Commentaires, $n^{\circ} 43$ et $n^{\circ} 44,1988$, p. 613-623 et 955-962; Raymond Boudon, «Effets pervers de la compassion», Commentaires, n¹17, 2007, p. 287-289.

3. On pourra certes objecter que de nombreux gouvernements actuels, en Europe comme aux Amériques, portés par une vague populiste et nationaliste, n'ont que faire de la compassion et marquent a contrario un durcissement de l'âme politique par l'élaboration de politiques migratoires répressives, l'érection d'obstacles à la traversée des frontières et à la mise en œuvre de procédures virulentes d'expulsion du territoire. Mais ce serait faire fi de l'indignation et de la mobilisation que ces pratiques politiques suscitent, marquant chaque fois la proximité avec ces êtres bafoués et maltraités comme un vecteur fort de la prise de parole publique. 
tion à l'autre, par nature dépendant, le nexus de la vie sociale 4 . Elle s'est inscrite également en droite ligne de la réception du récit victimaire comme mode de présentation de soi et à la demande de reconnaissance du statut de victime $^{5}$, faisant de la souffrance sociale une modalité d'expression de la difficulté à vivre en société en même temps qu'un nouveau paradigme de l'intervention sociale ${ }^{6}$. Elle a enfin été amplifiée par la médiatisation exacerbée des catastrophes (naturelles ou humaines) et les appels démultipliés à la bonne volonté (téléthon, cagnotte en ligne, souscriptions et dons en tout genre...) qui en ont fait un ressort émotionnel d'action sur les (bonnes) consciences ${ }^{7}$.

Deux grandes explications sont régulièrement mobilisées pour rendre compte de cette prégnance de la compassion dans les sociétés contemporaines. La première, fortement marquée par les intuitions séminales d'Alexis de Tocqueville dans De la Démocratie en Amérique ${ }^{8}$, est emblématiquement portée aujourd'hui par Myriam Revault d'Allones'. Elle lie la montée de la compassion aux caractéristiques par essence de la démocratie moderne. Le postulat d'égalité formelle qui la supporte et le processus d'égalisation des conditions qui l'anime développent un fort sentiment de ressemblance et donc une capacité à aisément ressentir ce qu'éprouve l'autre parce qu'il est un semblable. Cette reconnaissance du semblable dans le prochain, ici entendu comme le concitoyen, induit une logique de compassion étendue. En d'autres termes, la montée de l'égalitarisme a impliqué une valorisation croissante du souci pour autrui et nous a fait entrer dans une démocratie compassionnelle exacerbée par notre «passion identifiante» à la souffrance du semblable qu'est autrui.

La seconde, portée avec optimisme par Jeremy Rifkin ${ }^{10}$, part du constat que, volens nolens, désormais, les risques climatiques, nucléaires, industriels... sont communs à tous et à toutes, car ils produisent leurs effets sur la planète tout entière quand ils se concrétisent. Nous les partageons donc, parce que

4. Cf., à titre emblématique, Carol Gilligan, Une Voix différente. Pour une éthique du care, Paris, Flammarion, 2008 [1982]; Joan Tronto, Un Monde vulnérable. Pour une politique du care, Paris, La Découverte, 2009 [1993].

5. Voir Jean-Michel Chaumont, La Concurrence des victimes. Génocide, identité, reconnaissance, Paris, la Découverte, 1997; Guillaume Erner, La Société des victimes, Paris, la Découverte, 2006; et, tout récemment, Michel Messu, L'Ère de la victimisation, La Tour-d'Aigues, Éditions de l'Aube, 2018.

6. Didier Fassin a fort bien souligné les conditions de l'émergence de cette configuration sémantique compassionnelle dans le champ politique en lien avec une série de travaux issus des sciences sociales. Didier Fassin, «Souffrir par le social, gouverner par l'écoute», Politix, vol. 1, n 73, 2006, p.137-157. Voir aussi Jean Furtos et Christian Laval (dir.), La Santé mentale en actes, de la clinique au politique, Toulouse, Éditions Érès, 2005; Michel Joubert et Claude Louzoun (dir.), Répondre à la souffrance sociale, Toulouse, Éditions Érès, 2005; Marc-Henry Soulet (dir.), La Souffrance sociale, nouveau malaise dans la civilisation, Fribourg, Academic Press Fribourg, 2007.

7. Luc Boltanski a très tôt analysé ces formes d'engagement à distance. Luc Boltanski, La Souffrance à distance. Morale humanitaire, médias et politique, Paris, Éditions Métailié, 1993.

8. "Plus les hommes se sentent semblables les uns aux autres, plus ils sont sensibles à la misère d'autrui», Alexis de Tocqueville, De la démocratie en Amérique, Paris, Flammarion, 1981, p. 288.

9. Myriam Revault d'Allones, L'Homme compassionnel, Paris, Seuil, 2008.

10. Jeremy Rifkin, Une Nouvelle conscience pour un monde en crise. Vers une civilisation de l'empathie, Paris, Éditions Les liens qui libèrent, 2011 [2009]. 
nous sommes interdépendants en tant qu'êtres vivant dans un même espace, la Terre, et parce que nous sommes exposés aux mêmes risques. Malgré nous, donc, nous sommes concernés et affectés par ce qui touche tout membre de l'espèce humaine, parce que, en somme, ce qui va affecter celui qui est à l'autre bout du monde peut aussi nous atteindre ou nous faire subir un impact grandement dommageable. Pour Jeremy Rifkin, l'autre partageant la même condition malgré sa différence, c'est l'humain, le membre de l'espèce humaine vivant ou à venir ${ }^{11}$. Il ajoute toutefois, en prenant appui sur la World Values Survey pilotée par Ronald Inglehart ${ }^{12}$, que cette conscience d'être interdépendants n'est pas également partagée et qu'elle dépend fortement des conditions d'existence. En d'autres termes, le sens de l'empathie pour leurs congénères s'accroît avec le sentiment de sécurité ontologique dans lequel vivent les individus.

L'objectif de cette contribution à ce dossier sur la compassion n'est pas de statuer sur les manifestations que la compassion prend aujourd'hui dans le champ médiatique comme dans le champ politique, ni, encore moins, d'apporter une nouvelle pierre aux explications qui rendent compte de cet avènement. Pas plus qu'il ne s'agit de poursuivre la discussion sur les vertus politiques $^{13}$ et morales ${ }^{14}$ de la compassion.

11. «Nous assistons à une extraordinaire vague de solidarité mondiale, comme nous en avions déjà connu pour le tsunami de décembre 2004 et le séisme d'Haïti en janvier 2010. Un puissant sentiment d'inquiétude et d'altruisme soulève des centaines de millions de personnes autour du monde. Ce sont des exemples très forts de la nouvelle réalité empathique qui gagne l'humanité. Aujourd'hui, un drame collectif, une catastrophe écologique, un accident nucléaire touche chacun d'entre nous. Nous partageons les souffrances des autres, nous nous rendons compte qu'elles sont les nôtres, en nous identifiant à eux. Comment comprendre une telle empathie? D'abord, nous sommes concernés par ces drames car nous savons qu'ils pourraient aussi bien nous arriver, que ce qui affecte la biosphère là-bas nous affectera bientôt ici. Nous sommes sortis de l'ère égoïste de la fin du XXe siècle, nous nous découvrons tous reliés, interdépendants... » Jeremy Rifkin, «Une empathie nouvelle sur ce nouveau cosmopolitisme gagne l'humanité», entretien avec Frédéric Joignot, Le Monde Magazine, 15 avril 2011, p. 3.

12. Cf. Pippa Norris et Ronald Ingelhart, Cosmopolitan Communications: Cultural Diversity in a Globalized Word, New York, Cambridge University Press, 2009.

13. L'examen politique de la compassion, fortement marqué par la position d'Hannah Arendt, soulignant la différence entre compassion et pitié, explicite ce qu'est une politique de la pitié et identifie les effets négatifs qu'elle produit en termes de liberté. Hannah Arendt voit la compassion comme «la plus puissante et la plus dévastatrice de toutes les passions» et en fait le strict contraire de la liberté. Une telle lecture repousse la compassion dans la sphère privée afin d'expurger la politique de son contenu affectif. «La compassion... reste, politiquement parlant, impertinente et sans conséquence.» Hannah Arendt, Essai sur la Révolution, Paris, Gallimard, 2013, p. 81 [1963]. Cf. à ce sujet Sophie Bourgault, «Arendt, compassion et politique», Implicationphilosophiques.org. Thèse que reprennent et prolongent sous d'autres formes Marcel Gauchet et Myriam Revault d'Allones. Marcel Gauchet, «Les droits de l'homme ne sont pas une politique», Le Débat, vol. 3, n 3, 1980, p. 3-21; M. Revault d'Allones, L'Homme compassionnel, op. cit.

14. Le débat sur la valeur morale de la compassion ou sur celle de son analogon dans cette perspective, la sympathie, se demande de façon récurrente si, in fine, il s'agit d'un sentiment altruiste ou d'un calcul profondément égoïste. Avec ses défenseurs absolus (Jean-Jacques Rousseau, Arthur Schopenhauer, Emmanuel Levinas) et ses contempteurs radicaux (Les Cyniques, les Stoïciens, Bernard de Mandeville, Emmanuel Kant, Friedrich Nietzsche...). Cf. à ce sujet Christine Tappolet, "Compassion et altruisme», Studia Philosophica, n 59, 2000, p. 175-193. On se souvient de cette apostrophe sans concession sur la pitié (ici prise comme équivalent de la compassion) adressée par Bernard de Mandeville qui, si elle est, certes, «la plus belle et la moins nocive de nos passions, [elle] n'en est pas moins un défaut de notre nature tout autant que la colère ou l'orgueil » parce que les actions qu'elle enclenche sont portées 
Notre propos est bien davantage d'examiner ce que cette notion veut dire d'un point de vue sociologique, ce qu'elle nous apprend et permet de comprendre de l'activité sociale. Cet article vise en ce sens à identifier les vertus conceptuelles de la compassion, en dégageant ce qu'elle recouvre et ce à quoi elle engage à la lumière de deux variables centrales de l'analyse sociologique: d'une part, sa qualité de relation sociale et, d'autre part, son rapport à l'action, avec à l'esprit la volonté de comprendre comment, derrière l'affect qu'elle exprime, elle peut se transmuer, ou non, en force agissante.

\section{La compassion: une relation sociale particulière}

Cela étant énoncé, posons donc une première pierre dans cette perspective : tenter de comprendre sociologiquement la compassion, c'est la considérer d'abord comme une relation, c'est la saisir avant tout en tant que sentiment relationnel qui serait, toutefois, doté de propriétés particulières.

Il serait possible, pour ce faire, de partir de la relation sentimentale par excellence qu'est l'amour. Non pas en s'intéressant au choix du conjoint (on retrouverait les déterminismes sociaux), mais en s'intéressant à ce qu'est l'amour par nature (sociale bien sûr). Certes, on peut voir l'amour comme un absolu romantique gratuit (rien en échange), non forcément réciproque (pas payé en retour) et inconditionnel (ne dépendant pas du fait que quelque chose advienne). Mais pour peu qu'on l'envisage sous l'angle relationnel, sans pour autant tomber dans l'implacable logique bourdieusienne qui nous fait considérer qu'il s'agit d'une sorte de trêve miraculeuse où la domination est dominée ${ }^{15}$, force est d'admettre que nous avons à faire à une relation symétrique, à défaut d'être égalitaire, visant à produire un monde commun, une forme de coopération, idéalisée et faiblement visible comme telle, pour se représenter le monde et agir dessus ${ }^{16}$. Mais, là où la différence se marque par rapport à la compassion, c'est sur la symétrie qu'inclut l'amour, à partir du moment bien sûr où il est partagé, une propriété qui ne semble pas relever de la catégorie compassion puisque le point de départ de la compassion, c'est le fait qu'Autrui exprime de la souffrance et qu'Ego la ressente, et qu'en soit bannie toute idée de réciprocité.

Tournons-nous alors plutôt vers un autre sentiment relationnel, la honte, que Vincent de Gaulejac a fort bien analysé1 ${ }^{17}$. Nous sommes ici face à une relation fondamentalement asymétrique, face à un rapport social défavorable

par l'égoïsme puisqu'elles visent avant tout à soulager sa propre peine. Bernard de Mandeville, La Fable des abeilles, Paris, Éditions Pocket, 2017 [1714], p. 51.

15. Pierre Bourdieu, La Domination masculine, Paris, Seuil, 1998.

16. Francesco Alberoni, La Choc amoureux. Recherches sur l'état naissant de l'amour, Paris, Éditions Pocket, 1979.

17. Vincent De Gaulejac, Les Sources de la honte, Paris, Desclée de Brouwer, 1996. 
sur lequel on ne peut agir et qu'on doit endosser comme tel. Le sentiment de honte résulte justement de l'incorporation de ce rapport social. Dans cette asymétrie, celui qui éprouve le sentiment est «en bas» dans la relation.

Repartons donc de cette idée d'asymétrie, mais en la reconsidérant à la lumière des propriétés relationnelles de la compassion. Comme relation sociale, la compassion caractérise une asymétrie où celui qui éprouve le sentiment est «en haut» dans la relation; il est, en quelque sorte, au bénéfice de celle-ci puisqu'il souffre de la souffrance exprimée par autrui sans directement expérimenter cette souffrance. Philippe Corcuff appelle ainsi à considérer «une figure d'inversion de la dissymétrie des rapports entre deux catégories». Il appelle cette dissymétrie renversée le régime de la compassion ou le régime d'interpellation éthique dans le face à face, en faisant ainsi écho aux travaux d'Emmanuel Levinas. La compassion circonscrit, dit-il, «des moments de l'activité sociale où la dissymétrie du rapport dominant/dominé se trouve inversée, puisque le "dominant" tend à y être appelé au service du “dominé"18 ».

Comment dès lors comprendre qu'une telle configuration relationnelle produise un tel sentiment pour l'autre? Probablement par le fait que la compassion, c'est d'abord une relation imaginée ${ }^{19}$, i.e. du seul point de vue d'abord de celui-ci qui ressent, qui naît de la perception du malheur d'autrui. La compassion est une relation qui, in fine, n'est que relation, comme l'illustre l'exemple de la grand-mère d'Ari Gounongbé profondément préoccupée littéralement absente même parce qu'absorbée par l'autre souffrant. Elle lui explique ainsi, devant ses questions pressantes à comprendre l'objet de son profond souci, que «... seule la pensée qui exprime une préoccupation excessive et désespérée sert de lien et de soutien au souffrant ${ }^{20}{ }$ et ce, parce que le lien, s'il ne calme pas la douleur, abaisse le seuil de solitude que révèle la souffrance. Dire que la compassion est une relation qui ne vise que la relation peut être explicité en mobilisant trois registres analytiques.

18. Philippe Corcuff, «Ordre institutionnel, fluidité situationnelle et compassion. Les interactions au guichet de deux CAF», Recherches et Prévisions, $n^{\circ}$ 45, septembre 1996, p. 27.

19. Ce que soulignait déjà Georges Duhamel, cet écrivain médecin humaniste, dont toute l'œuvre est imprégnée par la compassion, en nous donnant à voir celle-ci comme une expérience médiate de la souffrance. "Voir souffrir les hommes, ce m'est odieux parce que mon imagination m'associe très péniblement à leur souffrance», Georges Duhamel, La Nuit d'orage, Paris, Le Club français du livre, 1947, p. XV. Déjà, considérer la compassion comme d'abord le fruit d'une représentation née de la vue du malheur d'autrui avait été souligné par Thomas Hobbes. Thomas Hobbes, De la nature humaine, Paris, Librairie Vrin, 1971.

20. A. Gounongbé, op. cit., p. 10. 


\section{La compassion égalise par la relation}

Non seulement elle réunit le souffrant et le compatissant, mais elle les rapproche. Quoique asymétrique, elle est un sentiment horizontal, à tout le moins horizontalisant comme l'exprime André Comte-Sponville. « ... elle n'a de sens qu'entre égaux, ou plutôt, et mieux, elle réalise cette égalité entre celui qui souffre et celui, à côté de lui et dès lors sur le même plan, qui partage sa souffrance ${ }^{21} »$. Eamonn Callan va même plus loin en nous expliquant ce qui est au principe de cette égalisation symbolique. Le destinataire de la compassion, à la différence de celui de la pitié qui est perçu comme submergé par son infortune, est pensé comme ayant maintenu son autonomie, d'où le fait de se sentir égal à lui et de le considérer comme un de ses pairs. «En montrant de la compassion pour autrui, j'affirme tacitement le statut commun que nous partageons en tant qu'êtres humains autonomes vulnérables à l'infortune; en montrant de la pitié, je désavoue ce statut commun ${ }^{22}$.» En ce sens, la compassion est un marqueur de reconnaissance, ne faisant pas de l'autre uniquement quelqu'un digne de notre attention, mais le rendant, à ses yeux comme aux nôtres, un égal parce que, comme nous, autonome ${ }^{23}$. La compassion est en ce sens intersubjective; autrui y est considéré comme un sujet et non comme un objet d'attention.

\section{La compassion refuse l'identification dans la relation}

Elle entend en effet rester une relation entre deux termes qui, quoique semblables, ne sont pas confondus. La non-confusion est, en premier lieu, une modalité de la reconnaissance de l'autre comme un semblable dissemblable (sinon cela signifierait que le compatissant a totalement absorbé le souffrant). Mais elle est, en second lieu, une condition de l'engagement dans une action rationnelle (sinon cela voudrait dire que le souffrant a complètement absorbé le compatissant). Ainsi, la compassion nécessite une claire démarcation entre les individus concernés. "Si quelqu'un a réellement fait l'expérience de ressentir la souffrance d'autrui dans son propre corps, alors il a précisément échoué à comprendre la souffrance de l'autre en tant qu'autre ${ }^{24}$.» Et cela, pour reprendre un argument d'Hannah Arendt, parce que l'espace mondain entre

21. André Comte-Sponville, Petit traité des grandes vertus, Paris, Seuil, 2001, p. 154.

22. Eamonn Callan, «The moral status of pity», Canadian Journal of Philosophy, vol. 18, n 1, 1988, p. 3. Traduction libre. La pitié comporterait pour lui un relent de mépris ou de condescendance, déniant en quelque sorte cette commune humanité qu'affiche la compassion.

23. La thèse d'Agata Zielinski repose sur un autre argumentaire mais parvient à la même conclusion d'égalisation. La compassion repose sur la reconnaissance de la vulnérabilité inhérente à la condition humaine permettant de «rencontrer autrui sans se tenir dans une position de surplomb, d'établir une certaine égalité dans l'asymétrie de la relation». Agata Zielinski, «La compassion, de l'affection à l'action», Études, n 4101, 2009, p. 61.

24. Martha Nussbaum, «Compassion, the basic social emotion», Social Philosophy and Policy, vol. 13, n 1, 1996, p. 35. Italiques de l'auteure.Traduction libre. Agata Zielinski va aussi dans ce sens. «Le degré initial de la recon- 
les hommes et les femmes, la pluralité qui permet justement de les différencier, est détruit ${ }^{25}$. Mais, en même temps, pour paraphraser Paul Bloom ${ }^{26}$, il est crucial, pour pouvoir répondre au devoir d'agir, de mettre en œuvre une compétence cognitive consistant à imaginer la souffrance de son congénère tout en s'en sentant distinct et donc en ne ressentant pas l'identique souffrance de la personne en détresse. Cela est particulièrement perceptible dans tout le travail de gestion de la bonne distance des professionnels fortement exposés structurellement à la souffrance et aux malheurs d'autrui. Donc, la compassion ne réduit pas autrui à sa souffrance en lui permettant de demeurer dans son intégralité et sa différence, i.e de demeurer, et donc de se tenir comme être humain, au-delà de sa douleur.

\section{La compassion atteste d'une attention à l'autre par la relation}

La compassion nous fait tendre vers autrui (ad tendere); cette tension de l'esprit que marque l'attention, c'est la sollicitude qui nous ébranle ${ }^{27}$. Elle est sollicitude, car elle n'engage pas tant, selon une éthique de la responsabilité, à entreprendre une action qu'à manifester une attention. Rappelant qu'en ce sens, la compassion s'inscrit dans une éthique de la sollicitude telle que développée par Carol Gilligan, Pierre Zaoui souligne qu'elle exige avant toute autre considération "d'approfondir la relation de sollicitant à sollicité [...] La relation singulière prime ici l'action impérative ${ }^{28}$.» «Là où la souffrance isole, ajoute Agata Zielinski, la compassion ouvre à la relation [...] L'action fondamentale à laquelle dispose la compassion, c'est la relation: la compassion met en relation ${ }^{29}$.» La compassion est lien parce qu'elle oriente vers la sollicitude avec la double face qui caractérise cette dernière, le souci et le soin, la préoccupation entière et le geste attentif (fortement repérable dans l'usage pluriel du mot "avoir des sollicitudes pour quelqu'un ", autrement dit exprimer des manifestations concrètes de soins attentionnés). La sollicitude, c'est "le versant actif de la compassion ${ }^{30}$ », c'est un soin plein de souci, i.e. une activité qui vise l'allègement de la souffrance, et donc la modification de la situation, mais aussi qui marque une inquiétude permanente et une absence de repos en raison d'un trouble né d'autrui.

naissance dans la compassion, écrit-elle, est ce qui me porte à voir en l'autre souffrant mon semblable»... mais «La compassion exclut - et doit exclure - l'identification», Agata Zielinski, op. cit., p. 58.

25. Hannah Arendt, La Condition de l'homme moderne, Paris, Calmann-Lévy, 1961 [1958], p. 52.

26. Paul Bloom, Against Empathy. The Case for Rational Compassion, Londres, Bodley Head, 2016.

27. Ann Van Sevenant nous rappelle que l'étymologie latine de sollicitude est marquée par l'idée d'ébranlement (solus entier et citare, exciter, mettre en mouvement). Ann Van Sevenant, Philosophie de la sollicitude, Paris, Librairie Vrin, 2011.

28. P. Zaoui, op. cit., p. 4

29. A. Zielinski, op. cit., p. 63.

30. Id. 
Pour résumer cette première pierre de notre tentative d'édification sociologique de la notion de compassion, essayons-nous à proposer un premier élément de définition de la compassion. Il s'agit d'un sentiment éprouvé appelant à prendre part à la peine, voire à la souffrance, d'un autre concret, et à souhaiter l'amélioration de son sort. La compassion, ainsi entendue, serait alors un sentiment relationnel asymétrique ressenti par celui qui voit - mais ne vit pas - la peine ou la souffrance, une relation à flux unique, une relation qui, paradoxalement, ne suppose pas dans un premier temps un autre terme, si ce n'est que comme matérialité d'un spectacle et qui est ensuite tendue vers cet autre parce que souffrant. Mais la qualifier de relation sociale asymétrique attentionnée non réciproque ne suffit pas; il faut aussi marquer que cette relation sociale singulière entretient un rapport particulier à l'action.

\section{La compassion, une relation paradoxale à l'action}

Repartons pour ce faire d'une définition préalable aisément partageable: la compassion peut être définie par le fait qu'elle comporte «une disposition à/ou un désir de soulager les peines d'autrui ${ }^{31}$ ». Mais ce postulat d'un rapport inconditionnel à l'action, pour consubstantiel qu'il soit, demeure en fait non discuté. Si, en effet, intrinsèquement, la compassion comporte une disposition à agir, jamais la question de la transformation de cette disposition en action n'est posée, comme s'il allait de soi, puisque disposition à agir il y a, que l'action est évidente et, donc, que cette disposition peut s'actualiser. Et c'est justement ce sur quoi il nous faut maintenant nous pencher en posant notre deuxième pierre: tenter de comprendre sociologiquement la compassion suppose de devoir aussi examiner le rapport particulier que cette relation sociale entretient à l'action: elle est en effet marquée du sceau de l'impuissance à transformer la situation pénible qui affecte autrui, l'objet de la compassion. C'est en somme l'incapacité à agir sur la situation pénible vécue par autrui qui explique l'éprouvé de la compassion. Essayons de décliner les différentes propriétés de ce rapport singulier à l'action qu'entretient la compassion.

\section{La compassion, une relation marquée du sceau de l'impuissance}

Revenons à l'étymologie pour éclairer cette thèse. Compassion peut être vu comme venant de cum avec et passio passion, peine, épreuve. Mais passio est formé à partir du supin, passum, de patior souffrir. Je subis la passion de l'extérieur (comme l'emploi religieux du terme passion l'a longtemps

31. Christine Tappolet, «Pitié», dans Monique Canto-Sperber (dir.), Dictionnaire d'éthique et de philosophie morale, Paris, Presses universitaires de France, 1996, p. 1142. 
exemplifié). Je subis avec - plus que je souffre avec -, en quelque sorte. Quelque chose s'impose à moi, à mon imagination, à mes sens, à ma raison, que je subis sans pouvoir faire autrement, sans pouvoir faire qu'il n'en soit pas ainsi. Ce que Philippe Corcuff rappelle - en utilisant d'ailleurs, pour qualifier cette dimension de la compassion, un vocabulaire qui pourrait être emprunté à la sorcellerie - par «le fait d'être "pris", en pratique et de manière non réfléchie, par un sentiment de responsabilité vis-à-vis de la détresse d'autrui ${ }^{32}$ ». C'est probablement pourquoi la compassion est sociologiquement plus intéressante que la miséricorde, la commisération ou la pitié; elle place en effet en son centre un problème d'action. La compassion, c'est quelque chose qui nous arrive malgré nous, elle nous saisit, et nous n'y pouvons rien, comme le rappelle Agata Zielinski.

Quelque chose fait irruption et rupture, m'empêche de poursuivre sur ma lancée, impose un détour à mon itinéraire, m'arrête ! [...] Ce saisissement que je n'ai pas cherché et qui s'impose à moi malgré moi [...] La compassion commence dans le fait d'être ainsi exposé à autrui. D'être exposé corps à corps, car c'est à ma vue, à mon oreille, à mes sens que se présente la détresse d'autrui ${ }^{33}$.

Dès lors, il n'est pas sans intérêt de considérer qu'une des dimensions centrales de la compassion se trouve dans le rapport entre pâtir et agir, retrouvant ainsi le raisonnement de René Descartes dans les Passions de l'âme qui opposait l'agent (celui par qui la chose arrive) au patient (celui qui endure, qui subit la chose $)^{34}$. Dans la compassion, fait socialement curieux, l'agent est le malheureux et le patient le compatissant. Elle place bien au centre de ses propriétés le rapport à l'action mais elle ne le situe pas, comme on l'imagine peut-être trop rapidement, dans le lien entre pâtir avec et détermination à agir, mais bien plutôt dans le lien entre subir avec et incapacité à agir. Il faut restituer pleinement la forme passive de la passion, du pâtir, et l'opposer à la forme active de l'agir (d'autant plus que nous y invite un redoublement étymologique puisque le terme supin lui-même vient de supinus, couché sur le dos, oisif, non actif, marquant ainsi un but qui sera atteint sous une forme passive). Elle est en ce sens fort éloignée de l'empathie (rogerienne tout au moins) en ce que celle-ci relève de la technique, de l'intentionnalité (essayer de ressentir la situation vécue par l'autre comme celui-ci la ressent en adoptant volontairement son point de vue). La compassion, elle, est nécessairement non réfléchie. Elle révèle une relation dans laquelle est reçu à la figure l'inadmissible d'une situation sociale (variable selon les individus, $c f$. la théorie des sentiments

32. P. Corcuff, «Ordre institutionnel, fluidité situationnelle et compassion. Les interactions au guichet de deux CAF», op. cit., p. 31. Mes italiques.

33. A. Zielinski, op. cit., p. 56.

34. René Descartes, Les Passions de l'âme, Paris, Presses universitaires de France, 1988 [1649]. 
moraux $^{35}$ ) et face auquel on ne peut agir, on ne peut que partager (par imagination, à distance...). La compassion donne à voir une peine médiate qui est d'autant plus ressentie qu'elle ne peut être agie.

Ego est frappé par le malheur d'autrui, qui lui offre en quelque sorte son malheur, et Ego ne peut pas rendre, i.e. modifier ce qui l'affecte, son malheur ou sa peine. Nous ne sommes pas ici face à ce qu'invoquent Alain Caillé et Philippe Chanial dans leur introduction au volume L'Amour des autres, compassion, care, humanitaire, une logique $d u d^{36}{ }^{36}$. La compassion ne se présente pas comme une vertu, comme un geste généreux de l'Homo donator $^{37}$, une marque indéfectible de la primauté du don sur toute autre propriété du lien social. Au contraire, la compassion exprime davantage l'impossibilité de rendre, l'incapacité de faire de l'action subie la mesure d'un contre-agir, soulignant que peut-être ce qui se joue plus fondamentalement dans le lien social, ce n'est pas tant le don ou l'intérêt, mais la puissance à agir (avec, contre, sur, peu importe). «Je souffre à cause de la souffrance de l'autre, rappelle Agata Zielinski, et peut-être de l'impuissance à y remé$\operatorname{dier}^{38}$.»

Certes, il faut entendre la souffrance de l'autre pour pouvoir aider, mais on n'agit pas en la prenant avec soi, en l'endossant. La compassion ne comporte pas l'action, non seulement elle ne se transforme pas en production d'un agir - sauf à souhaiter le soulagement d'un spectacle horrible, en y apportant bien souvent la méthode du général Potemkine - elle en est au contraire l'antithèse, le strict opposé. La compassion est la marque de l'impuissance appelée à se perpétuer dans le cercle vertueux de l'inadmissible, de l'impuissance, de la compassion, puis de l'inadmissible... Ce que nous indique la compassion, derrière l'impérieux désir d'agir que l'épreuve de la souffrance d'autrui nous impose, c'est l'impossibilité de toute action, de quelque nature qu'elle soit, sinon, depuis longtemps, une action aurait été engagée pour remédier à une situation si insupportable. Ce que nous rappelait à sa façon Cicéron.

Plutôt que de plaindre les gens, pourquoi ne pas les secourir, si on le peut? $\mathrm{Ne}$ pouvons-nous être généreux sans éprouver de la pitié? Nous ne sommes pas tenus à prendre pour nous les chagrins des autres; mais, si nous le pouvons, à soulager les autres de leur chagrin ${ }^{39}$.

35. Adam Smith, Théorie des sentiments moraux, Plan-de-Tour, Éditions d'Aujourd'hui, 1982.

36. Alain Caillé et Philippe Chanial, «Présentation», Revue du Mauss, numéro spécial «L'amour des autres. Care, compassion et humanitarisme», $n^{\circ} 32,2008$.

37. Jacques T. Godbout, Le Don, la dette et l'identité: homo donator versus homo oeconomicus, Paris, la Découverte, 2000.

38. A. Zielinski, op. cit., p. 60. Italiques de l'auteure.

39. Cicéron, Tusculanes, IV, 26, Paris, Édition La Pléiade, 1962, p. 350. 


\section{La compassion, une manifestation d'impuissance appelant l'action}

Deux grands pôles de la compassion: partage imaginé de la souffrance et aide souhaitée impérativement donc, affliction médiate par transport d'expérience et obligation morale d'agir en quelque sorte. Mais là encore, si l'obligation morale renvoie à l'idée d'action, elle n'est toutefois pas action. La compassion naît d'un abattement de l'esprit devant l'éprouvant d'un spectacle, en même temps que du constat de ne pas savoir quoi faire malgré le sentiment de devoir agir. La compassion appelle l'action, elle ne produit pas l'action; elle est porteuse d'une volonté d'agir, elle n'est pas productrice de l'action. En ce sens, on peut dire que la compassion est un support de l'action. «Elle met l'être en mouvement, elle est une réaction qui fait agir ${ }^{40}$.»

Elle donne certes l'impulsion première à l'action pour autrui, mais elle n'est pas en soi active; elle n'est qu'un désir d'agir, pour reprendre la définition de Christine Tappolet, élément de définition que reprend Ari Gounongbé en faisant de la compassion une émotion ressentie à la vue de la douleur des autres qui a pour spécificité d'induire chez soi un désir d'alléger la douleur ou d'enlever la cause ${ }^{41}$. Elle n'entraîne donc pas «une réaction de solidarité active»; elle l'appelle ${ }^{42}$ et libère des volontés d'agir. Mais elle n'existe que tant que ladite réaction ne se concrétise pas. Elle porte à venir en aide à l'autre souffrant, mais elle n'est pas elle-même l'aide apportée à cet autre souffrant. Certes, elle est au cœur de la motivation morale et donne l'impulsion première à l'action désintéressée pour les autres, mais elle n'est pas cette action parce qu'elle est par essence passive tant elle nait justement de la perception de l'impuissance à agir. Si elle était cette action, soit elle n'aurait pas été compassion (il n'aurait pas été nécessaire de compatir, agir aurait suffi), soit elle ne serait plus compassion parce qu'elle aurait dépassé cette impuissance momentanée. Il est en ce sens vital, pour apprécier pleinement ce que recouvre la notion même de compassion, de comprendre

40. Zona Zaric, «Pourquoi avons-nous besoin de la compassion?», IPhilo, la philo en poche http://iphio.fr, 27 mai 2017, p.2

41. A. Gounongbé, op. cit., p. 14

42. Je me situe ici en opposition à la thèse formulée par Jean-François David (La Compassion dans l'exercice professionnel du travailleur social, Mémoire de Diplôme supérieur en travail social, Metz, Institut régional du travail social de Lorraine, 2008) quand il avance que la compassion est un régime d'action dans le champ du travail social permettant de faire son travail (p. 16), une compétence morale en situation professionnelle ouvrant des enjeux en termes de reconnaissance et de valorisation du travail d'accompagnement, un savoirfaire professionnel parmi d'autres auquel le travailleur social peut recourir. La compassion, en tant que régime d'action, serait alors l'aspect peu visible d'une dimension plus générale du travail social - le care -, à réintégrer dans le champ des activités sociales comme un pan négligé de l'activité professionnelle (p. 23). Pour justifier son propos, il réfère à Philippe Corcuff. Mais ce dernier ne parle pas de la compassion comme d'un régime d'action; il la voit bien davantage comme un régime d'engagement dans l'action. P. Corcuff, «Justification, stratégie et compassion: Apport de la sociologie des régimes d'action», op. cit. Le régime de compassion, c'est en ce sens un mode d'engagement spécifique dans l'action, et non un mode spécifique d'action. 
la distinction de ce qui appelle l'aide et de ce qui la concrétise. Dès qu'il y a action, la compassion n'est plus, elle devient autre chose: engagement, mobilisation, indignation... Elle n'est que le moment d'une impuissance devant cette souffrance qui affecte et dont il est impossible de se détourner. Elle est certes obligation à faire mais elle est en même temps la conséquence de l'impossibilité de faire. Sinon, elle se transmue.

\section{La compassion, une relation momentanée de reconnaissance d'autrui}

La compassion est une relation momentanée. Maintenant, hic et nunc. Dans la compassion, il n'y a pas de projet possible, pas de pro-jection. On est enfermé dans le présent de la situation: présentisme et hypertrophie de l'instant. Dès qu'on en sort, il y a action. Et si on n'en sort pas, si le moment compassionnel dure, si l'impuissance s'enracine, alors émergent apitoiement ou détournement. Mais, ce faisant, la compassion est une relation de reconnaissance d'autrui. Si la compassion n'engage pas, elle oblige par contre. Elle est en ce sens une dette anthropologique. Nous sommes les obligés de l'être souffrant. Nous lui devons quelque chose. Nous lui devons un regard, au moins, celui qui reconnaît son malheur.

C'est en cela que la compassion est plus qu'un sentiment, plus qu'une bonne conscience, plus qu'un flux à sens unique. Parce qu'il y a interpellation, il y a obligation morale d'interaction. Parce qu'il y a considération, il y a souci d'échange. Parce qu'il y a interpellation, il y a devoir moral et social. La compassion est en ce sens un accusé de réception d'un souci de partage et, ce faisant, le marqueur d'une volonté de reconnaissance de l'autre souffrant. Et si allègement de la souffrance il y a eu, si un réconfort est advenu, ce n'est pas en raison de l'efficacité d'une quelconque action concrète, mais bien parce que, malgré la vanité de toute action, quelque chose s'est passé, aussi furtif ait-il été. Indéniablement, elle est un élan vers autrui qui, s’il n'est pas une action, s'il n'est qu'un geste, est un geste signifiant. C'est bien pour cela que le simple fait d'éprouver de la compassion pour la souffrance d'autrui allège ses souffrances quand bien même, paradoxalement, cela ne change pas sa situation, et cela même si aucune action n'est entreprise pour le soulager. Entendre la souffrance d'autrui et le lui signifier ouvrent la porte à une demande d'attention. Je compatis quand je reconnais tout comme je reconnais quand je compatis. J'admets ce que je n'avais pas vu avant et, en reconnaissant, je confirme que j'ai vu la souffrance mais je n'accole pas à cette objectivation de la situation douloureuse que connaît autrui, une quelconque option de pouvoir agir, simplement le souhait, le désir, de voir la situation transformée. Il y a un abîme en ce sens entre compassion et action 
que ne vient pas obturer la reconnaissance; celle-ci vient simplement ouvrir la brèche de la nécessité/de la légitimité/du désir, à partir du regret qu'il en soit ainsi, de voir (et non de faire) advenir autre chose, naître une situation autre. Malgré ainsi son impuissance constitutive, la compassion est actualisation d'un lien, elle est marqueur ou rappel d'une commune appartenance en même temps qu'expression d'une émotion. La compassion, le temps que dure le spectacle, réunit des étrangers et les mue en frères humains, lointains mais réels. Elle implique un sentiment d'humanité partagée, ce à quoi pensait Arthur Schopenhauer en voyant dans la Mitleid, l'équivalent germanique de la compassion, la manifestation d'une commune appartenance. Nous ne pouvons compatir qu'aux souffrances de ceux pour qui nous n'éprouvons aucune indifférence, nous rappelle Aristote. La compassion repose en ce sens sur la philia, pas tant sur l'amour en tant que tel, mais sur le fait de considérer autrui comme un être aimable, de le reconnaître donc comme semblable et comme proche, et non comme un barbare ${ }^{43}$.

\section{Les origines de l'impuissance à agir dans la compassion}

Reste à comprendre d'où provient cette impuissance à agir qui est attachée à la compassion, à en expliquer l'origine et à en dégager les fondements. Probablement faut-il chercher la réponse dans l'entrelacs de plusieurs registres et, dès lors, considérer cette impuissance à agir comme la résultante de plusieurs effets qui jouent à des degrés divers et se combinent en des modalités variables selon les configurations.

\section{Premier effet: le tragique ou la vacuité de toute action}

Pour tenter de comprendre ce premier registre pouvant rendre compte de l'origine de l'impuissance à agir constitutive de la compassion, un détour par la tragédie, au sens théâtral du terme, peut être un recours. Pour ce faire, revenons quelques instants à Antigone, dont la version théâtrale de Jean Anouilh illustre le plus clairement le paradoxe tragique de la compassion: le souci jusqu'à l'absurde de l'autre lorsque Créon apprend à Antigone que les corps de Polynice et d'Étéocle étaient un tel amas de chair qu'il a arbitrairement décidé qu'une partie serait affectée au premier et une autre au second. Forcée par le devoir sacré d'ensevelir l'âme du premier afin qu'elle n'erre pas définitivement, et ce, malgré l'interdiction de Créon, Antigone fait pitié à vouloir ce qui est impossible. Et Créon en a pitié. C'est empli de compassion

43. Bien sûr, les principes sur lesquels reposent la proximité et la similitude ne sont pas aujourd'hui du même ordre qu'à l'époque de la Cité grecque. Paul Audi, «D’une compassion à l'autre», Revue du MAUSS, numéro spécial «L'amour des autres: care, compassion et humanitarisme», op. cit., p.185-202. 
pour elle qu'il lui propose un vertueux mensonge pour éviter le pire, qu'il lui propose un petit bonheur ordinaire avec Hémon au prix de l'oubli de son acte. Mais Antigone refuse et lui offre sa mort, l'enjoint de tenir sa promesse (de tuer celui qui ensevelira Polynice). «Je l'ai forcé, lui et ses hommes, à me devenir secourable», lui fait dire Henri Bauchau ${ }^{44}$. Créon ne peut agir, il est littéralement en état d'a-crasie, lui le chef d'État, tenu par cette mort qui lui est offerte. Il n'a d'autre possibilité d'agir que de laisser faire ce qui devait se faire, contre son impulsion première, contre son émotion profonde, malgré aussi le partage qu'il a de la souffrance d'Antigone. Sa compassion est la preuve de son impuissance devant cette situation et le condamne à tenir sa promesse. Antigone l'oblige, comme le malheur de ces semblables lointains nous oblige à partager leur peine, en souhaitant qu'il n'en soit pas ainsi. Seulement, comme pour Créon face à Antigone, la compassion nous rappelle l'impossibilité de toute action face à ce pâtir de l'autre que nous subissons de plein fouet, nous rappelle la vacuité de toute action prise à partir des présupposés mêmes de la compassion.

La tragédie, ce n'est pas le drame traversé par des risques, des dangers, des épreuves mais dont le jeu est ouvert et dans lequel les acteurs peuvent influer sur la fin par les actions qu'ils conduisent. La tragédie, au contraire, est un enchaînement d'événements funestes dont l'issue est fatale, implacable ${ }^{45}$. Le résultat est connu à l'avance. On n'y peut rien, ni les acteurs ni les spectateurs. Simplement laisser le spectacle se dérouler sous nos yeux et, au mieux, partager à distance le destin des acteurs, le comprendre i.e. le prendre avec soi, le temps de la pièce, le temps du spectacle, le temps de l'exposition. Comment en effet avoir de l'espoir, avoir même simplement l'idée de changer ce qui est immuable et fatal? Fatal comme le destin, extérieur à la volonté humaine qui régit nos vies en fixant de façon irrévocable le cours des événements, qui s'impose à nous indépendamment de notre volonté.

Il n'y a en ce sens pas d'espoir dans la compassion. C'est peut-être pour cela que nous sommes davantage compatissants devant les victimes de catastrophes naturelles que devant les victimes de l'exploitation humaine. Pour les secondes, elles comme nous aurions pu, aurions dû, pourrions encore, devrions toujours, faire quelque chose ${ }^{46}$. Changer la situation, intervenir dans l'ordre des choses. Bref la situation est transformable; c'est une affaire d'éner-

44. Henri Bauchau, La Lumière Antigone, Arles, Éditions Actes Sud, 2009, p. 15.

45. «C'est cela qui est commode dans la tragédie [...] c'est minutieux, bien huilé depuis toujours [...] c'est propre la tragédie. C'est reposant, c'est sûr [...] Parce qu'on sait qu'il n'y a plus d'espoir», Jean Anouilh, Antigone, Paris, Éditions de la table ronde, 1946, p. 53-54.

46. «La différence entre infortune et injustice, rappelle la politologue américaine Judith Shklar, met le plus souvent en jeu notre volonté aussi bien que notre capacité d'agir ou non en faveur des victimes, de condamner ou d'absoudre, d'apporter de l'aide, d'atténuer les souffrances et d'offrir des compensations ou bien tout au contraire de détourner notre attention», Judith Shklar, Visages de l'injustice, Belfort, Éditions Circé, 2002, p. 10. 
gie, de volonté, de mobilisation, de lutte. On ne compatit pas avec les ouvriers quand on a accédé à l'intelligence du mouvement historique dont est porteur le prolétariat. On combat avec eux! Et ce qui vaut pour la classe ouvrière, vaut pour les femmes, pour les peuples autochtones, pour les enfants travailleurs, pour tous les opprimés de la terre. Pour les victimes de catastrophes naturelles, par contre, le malheur a frappé, imprévisible et implacable; il a frappé les bons comme les mauvais, indistinctement. Les victimes n'y peuvent rien, ne peuvent rien contre ce fléau qui les anéantit. Elles n'ont pu l'éviter car on ne peut se prémunir du mauvais sort. C'est au mieux une conjonction maligne et néfaste des éléments, au pire un avertissement des Dieux. Comme la peste qui frappe Thèbes et oblige Edipe à chercher, funeste quête, le meurtrier de Laïos. Nous, les spectateurs, nous n'y pouvons rien non plus. Même pas réparer, à peine alléger - $c f$. comme illustration, disons la prochaine catastrophe naturelle qui fera le buzz demain sur nos supports médiatiques - tant l'aide peut être difficile, problématique, voire contre-productive parfois. Nous pouvons simplement témoigner de notre affectation devant ce malheur qui nous rend impuissants. Que faire en effet? Rien d'autre que de l'infiniment petit. Rien d'autre que de l'insignifiant et c'est cela qui nous rend d'autant plus sensibles à l'affliction qui frappe autrui.

En ce sens, c'est par un effet du tragique que la compassion relie des impuissants, ceux qui souffrent et ceux qui souffrent de voir des êtres souffrir. Toute action s'avérerait insensée puisque nous ne pouvons avoir prise sur la réalité dans la mesure où tout est déjà joué, puisque notre action ne parviendrait pas à contrer les forces agissantes productrices de la souffrance. Toute action serait vaine : nous sommes trop loin, nous arriverions trop tard, les forces à contrer sont trop pesantes, les déterminations sont trop lourdes, la situation est trop inextricable... Il n'y a pas, en l'occurrence, d'espoir à avoir parce que le destin a frappé et que nous ne pouvons rien contre la fatalité.

\section{Deuxième effet: le spectaculaire ou la dissolution de l'action}

La compassion renvoie à l'idée de s'émouvoir du spectacle de la souffrance d'un tiers qui ne la méritait pas. L'important ici, tout d'abord, par-delà l'émotion, c'est le spectacle, ce qu'Aristote soulignait déjà dans son ouvrage Rhétorique en disant que la pitié (eleos) - ici à entendre comme compassion-caractérisait une forme de souffrance née du spectacle du malheur d'autrui. La compassion est une double relation à la souffrance: d'une part, elle marque notre concernement à la souffrance de quelqu'un; d'autre part, elle souligne notre éloignement de cette souffrance puisque nous n'avons pas 
une expérience directe mais seulement un rapport médiat par l'entremise de la souffrance exprimée par autrui ${ }^{47}$. Elle nous est étrangère en même temps qu'accessible par un dispositif de mise en vue, d'où l'idée qu'il n'y a pas compassion sans spectacle de la souffrance d'un autrui. Et si le spectacle est insupportable, c'est bien d'abord parce que je ne peux m'y soustraire. C'est donc par le regard que je suis ému, et non par l'entendement. Le spectacle, c'est d'abord ce qui attire le regard malgré moi et donc qui est, toujours malgré moi, à mon corps défendant, susceptible d'éveiller des émotions en moi. La souffrance se donne en spectacle, s'expose à mon regard, s'offre à moi. La compassion en ce sens me prend par surprise. Je ne la cherche pas, elle me saisit. Ensuite, la compassion n'est légitime que si la souffrance est illégitime. Émerge de cette signification originelle l'idée d'une souffrance indue avec là deux hypothèses à creuser. Avons-nous à faire à un malheureux méritant, comme il y a des pauvres méritants ${ }^{48}$ ? Ce serait donc une forme d'injustice qui se loverait derrière la compassion que l'on ne saurait ressentir pour celui qui a bien cherché son sort. On ne saurait ainsi avoir de la compassion pour Antigone (qui d'ailleurs la refuse quand Créon esquisse ce registre). On ne peut avoir de compassion pour qui brave le destin et va contre le cours des choses. Il faut en quelque sorte faire montre d'un peu d'humilité pour susciter de la compassion. Pas de compassion pour les orgueilleux donc! Sommes-nous, au contraire, en présence d'un ressenti pour l'autre souffrant qui s'ancre dans le fait que je peux me sentir/croire susceptible d'être exposé un jour à un tel malheur? Comme Henri Bergson le rappelle, la crainte entre pour quelque chose dans la compassion que les maux d'autrui nous inspirent ${ }^{49}$. Je compatis alors par empathie anticipatrice et par inversion possible des situations. Pouvoir me penser dans cette situation, alors que je ne l'aurais (bien sûr) pas mérité me rend compréhensif. Je ne me mets pas à la place de l'autre, mais j'imagine que j'aurais pu ou je pourrais l'être ${ }^{50}$.

De surcroît, il n'y a pas de coupables, même s'il y a des victimes. Pas coupables les victimes, elles ne sont que le jouet du sort funeste, de la mal-

47. Fabienne Brugères, «Le spectacle de la souffrance peut-il être moral?», Raison publique, n 10, mai 2009. «S'il n'y a pas spectacle, surenchérit Pierre Zaoui, si l'on connaît par exemple le même sort que l'affligé, on n'éprouvera pas de la pitié, mais de la solidarité ou de l'égoïsme. La pitié exige que la souffrance soit donnée à voir à celui qui ne souffre pas», Pierre Zaoui, «De la pitié à la sollicitude», Vacarmes, n 33, octobre 2015, p. 2.

48. Ce que soulignait déjà Aristote: «Ce qui suscite la compassion, c'est tout ce qui est douloureux et destructeur - et particulièrement les maux dont le sort est la cause, c'est-à-dire ceux qui, en plus d'être immérités, sont humainement injustifiables. » Aristote, Rhétorique, II, Chap. VIII, Paris, Éditions Press Pocket, 2007, 86a. II y a de l'honnêteté à éprouver de la pitié (analogue ici à la compassion) «car sur ceux qui sont malheureux sans le mériter, on se doit affliger et apitoyer.» (Rhétorique, 86b).

49. Henri Bergson, Essai sur les données immédiates de la conscience, Paris, Éditions François Alcan, 1906, p. 27.

50. Je ne peux ainsi compatir à la situation dégradante faite à l'étranger dans mon pays que si je me sais ou je me pense voyageur, donc susceptible d'éprouver cette réalité (même si je ne vais jamais bouger de chez moi). Si je me pense comme un natif à l'identité locale chevillée au corps, même si je suis amené à voyager un jour, je ne peux pas comprendre l'expérience de l'humiliation de l'étranger, donc je ne compatis pas. 
chance, de la mal-heure. Être né là où on est né, se trouver à cet endroit-là et à ce moment-là. Edipe était l'instrument des Dieux et c'est en voulant fuir la malédiction qu'il l'a réalisée. Pas coupables non plus les spectateurs, ils ne sont pas responsables, ils sont même condamnés à voir le spectacle du monde. Lui aussi, si on veut le fuir, il nous rattrape. Ni responsables ni coupables, mais émus parce que la souffrance des malheureux ne laisse pas indifférent. Ce n'est pas parce qu'on est impuissant qu'on est indifférent. Au contraire même. Nous sommes émus parce qu'impuissants. Nous sommes empreints de compassion avec compassement, avec affectation et avec retenue. Comme lors d'un deuil. Ce deuil n'est pas le mien, le mort n'appartient pas au cercle de mes proches (sinon je serais celui qui souffre), mais je comprends le deuil et c'est cela que je veux dire quand je présente mes condoléances, ce témoignage que je fais à l'autre de la part que je prends à sa douleur, sans pour autant l'endosser.

L'impuissance à agir naît donc d'un effet de spectacularisation, au sens premier du terme ${ }^{51}$. L'émoi que suscite en nous la détresse de l'autre et l'attention vers lui qu'elle nous impose condensent dès lors la totalité de notre disponibilité, subjugués que nous sommes par la puissance émotionnelle du spectacle dont nous avons été saisis et qui nous saisit. Le spectacle nous rive devant l'obscène, i.e. devant ce qui ne peut être montré publiquement tant cela contrevient aux règles de la bienséance sociale; il nous cloue devant ce que nous ne devrions pas voir. Il est en ce sens paralysant et étouffe notre capacité d'action. À tout le moins, il nous oblige à réagir en nous invitant à la compassion qui dès lors devient l'action elle-même; l'action se dissout en ce sens dans la compassion, ce qu'exprime très fortement Pierre Zaoui, «Pour que la pitié devienne sentiment indistinctement moral et politique, il faut qu'elle se stimule dans un discours qui soutienne l'identification avec celui qui souffre en effaçant la distance du spectacle et ainsi en tenant lieu d'action ${ }^{52}$.»

51. Et bien souvent, aussi d'un effet d'esthétisation. C'est parce que la souffrance de l'autre est belle - et qu'elle n'est pas nôtre, sinon elle ne serait pas belle - que nous sommes touchés, que nous avons de la sympathie pour elle, et donc de la compassion pour l'autre. Parmi les modalités par lesquelles le spectacle de la souffrance se mue en parole agissante, Luc Bolstanski a ainsi identifié, à côté de la topique de la dénonciation et de celle du sentiment, la topique esthétique; il a fait de l'esthétisation de la souffrance un des ressorts du désir d'action pour l'autre souffrant et empêché. En ce sens, la souffrance ne saurait être silencieuse, et la compassion ne saurait naître d'une souffrance qui pourrait être supposée sans être vue, et encore moins visible. Luc Boltanski, op. cit. Il est possible, toutefois, de pousser son raisonnement et de voir également dans cette esthétisation un élément d'inhibition de la possibilité d'agir, sans pour autant faire de la beauté de la souffrance un grandissement de celui qui souffre.

52. Id. Mes italiques. 


\section{Troisième effet: le souci ou le substitut à l'action}

La compassion relie des êtres singuliers. Pour Hannah Arendt, dans Essai sur la révolution ${ }^{53}$, la compassion ne peut être ressentie pour une classe ou pour un peuple entier, son objet ne peut être qu'un individu spécifique, car, comme toute passion, elle ne peut comprendre que le particulier, elle ne peut être ressentie que pour un seul individu singulier alors que la pitié peut avoir comme objet une multitude. Considération que reprend aussi Luc Boltanski ${ }^{54}$, pour qui la compassion rencontre toujours des obstacles à sa généralisation et s'adresse à des êtres souffrants singuliers sans développer des capacités de généralisation qui lui permettraient de participer à une véritable politique humanitaire. En ce sens, je suis compatissant avec un être qui souffre, ce qu'ont très bien compris les ONG dans leur campagne de publicité en vue de la recherche de fonds quand elles mettent en scène des figures individuelles qui subsument la souffrance, comme l'a emblématiquement illustré la photographie de celle qu'on a nommée la Pietà algérienne (appelée aussi la Madone de Bentalha), symbole international du malheur aveugle et de la douleur incommensurable. Je ne peux compatir avec des anonymes, avec un groupe, encore moins avec une cause. La compassion est une relation d'être à être. Je m'associe aux intérêts d'un groupe, j'adhère à une cause, mais je compatis à la souffrance incarnée dans un corps, dans un regard. La compassion disjoint, fragmente, dissocie dans son principe même. Elle vise l'être humain dans sa fragilité malheureuse, pas l'être social dans ses statut et condition. La relation est d'emblée interhumaine. Point de solidarité avec une classe, mais au contraire une relation avec son prochain. Point d'engagement, mais avant tout une attention vers un autrui singulier.

On retrouve ici une intuition fondatrice d'Hannah Arendt, toujours dans Essais sur la révolution. La compassion est muette et passive, elle autorise des cris et des gestes simplement, pas des paroles et des actions ${ }^{55}$. Il s'agit d'une passion «muette» $(a$-logos $)$; elle est incapable de se traduire en mots. Elle est donc opposée à la parole et, par voie de conséquence, au dialogue qui sont eux absolument essentiels pour l'action. La compassion est, en d'autres

53. Hannah Arendt, Essai sur la révolution, Paris, Gallimard, 1967 [1963].

54. L. Boltanski, op. cit. Philippe Corcuff reprend cette même idée en énonçant qu'«avec la compassion, les difficultés d'autrui sont saisies et ressenties dans leur singularité». Philippe Corcuff, «Justification, stratégie et compassion: Apport de la sociologie des régimes d'action », Correspondances, bulletin d'information scientifique de l'IRMC, Tunis, $n^{\circ} 51$, juin 1998, p. 6.

55. Comme l'illustre la romancière Annie Ernaux au sujet de sa mère atteinte de la maladie d'Alzheimer. «Au moment de partir (de l'hôpital), je la reconduis à la salle à manger (j'allais écrire réfectoire comme au pensionnat). Une soignante lui donne un bonbon avec un joli sourire: "Prends, ça fait passer le temps". La compassion, pure.» Annie Ernaux, Je ne suis pas sortie de ma nuit, Paris, Gallimard, 1997, p. 59. 
termes, une notion mutique, tout juste bonne à produire des sons (et non des paroles/discours) et à susciter des gestes (pas des actions).

D'une certaine façon, en ce sens, en même temps que la compassion appelle un changement devant l'insupportable, elle est un sentiment conservateur. La souffrance ne peut être combattue, même pas allégée, simplement partagée... un instant, le temps que dure le spectacle. Qu'y a-t-il, au-delà, après la compassion? Rien. Ce n'est qu'un sentiment temporaire, fugace présent ici et «main-tenant», i.e. pendant que l'on tient quelque chose dans la main, comme le spécifie l'étymologie. Ainsi, la compassion ne nous engage pas durablement. Elle ne nous engage pas du tout d'ailleurs, si l'on reprend la définition que donne Jean Ladrière de l'engagement. Nous n'aliénons pas une partie de nous-mêmes pour la remettre entre les mains d'autrui, pour nous lier à son sort comme dans la relation amoureuse ou comme dans l'engagement militant ${ }^{56}$.

La compassion n'est pas en ce sens une action politique visant la transformation de la situation ${ }^{57}$, mais une action qui travaille la souffrance par son impuissance même, en se faisant partage et accompagnement, comme l'illustre l'exemple de l'accompagnement palliatif dans le travail social ${ }^{58}$. Elle est geste et attention et, par cela même, elle n'est pas politiquement signifiante. Elle disperse le politique, antithétique qu'elle est à un objectif de lutte contre un adversaire ou de mobilisation pour une cause et, dès lors, se disperse en actes, en émois, en signes, en gesticulations, en cris, en pleurs, en souci, en réconfort. Par cet effet de souci, la compassion se condense dans la relation parce que la relation prime sur l'action; elle dispense de l'action, au sens arendtien du terme ${ }^{59}$, elle est un renoncement stratégique à agir. Elle n'est

56. Jean Ladrière, «Engagement », Encyclopedia Universalis, 1990.

57. Comme le rappelle Myriam Revault d'Allonnes: «Soutenir que la compassion est le ressort anthropologique de la reconnaissance du semblable, ce n'est pas pour autant lui donner une pertinence politique», L'Homme compassionnel, op. cit., p. 102-103.

58. Au cœur de la dimension palliative du travail social, on retrouve quelque chose qui s'apparente à de la compassion pour qualifier une obligation d'agir sans mouvement, sans possibilité de projeter, et dont la qualité première est la dimension diplomatique, visant en priorité le maintien de la relation en espérant un réchauffement, i.e. l'émergence d'une possibilité d'agir. Marc-Henry Soulet, «La reconnaissance du travail social palliatif», Dépendances, n³3, 2007, p. 33-44. En conséquence, dans l'accompagnement social palliatif, «les qualités requises pour être "accompagnant" se fondent non plus tellement sur des exigences de justice, mais sur des exigences de justesse. L'empathie, la bonne distance, l'auto-réfléxivité sont autant d'outils qui se doivent d'être mobilisés dans la démarche d'accompagnement pour permettre l'établissement d'un lien reliant deux individus dont l'une des missions centrales est, dès lors, de se reconnaître mutuellement.» Yves Papadaniel, «La double négociation», Pensée plurielle, vol. 3, n²2, 2009, p. 8. Italiques de l'auteur.

59. On comprend mieux ainsi pourquoi, s'il n'est pas possible, pour le travailleur social, d'intervenir sans un sentiment de responsabilité vis-à-vis de la détresse d'autrui, son activité, par contre, consiste bien souvent, afin de pouvoir littéralement intervenir, à transformer un tort - au sens de Jean-François Lyotard (i.e. un dommage que l'on n'arrive pas à faire reconnaître pour la raison même qu'il s'accompagne de la perte des moyens de faire la preuve de son existence cf. Le Différend, Paris, Éditions de Minuit, 1983) - en une injustice, c'est-à-dire à donner prise à une capacité à le traiter socialement. Le travailleur social se doit ici d'aider l'usager à transformer son infortune en une situation qui peut être saisie par le droit ou donner lieu à des droits. 
pas intervention parce qu'elle ne peut inter-venir, enfermée qu'elle est dans la dualité de la relation. Mais plus qu'une excuse au non-engagement, elle en est une alternative, en quelque sorte.

\section{Quatrième effet: l'effondrement ou l'usure de la capacité à agir}

La compassion est usante. Elle affaiblit ${ }^{60}$. La compassion nous saisit et nous affecte, mais elle suppose son dépassement, sinon elle devient un puits sans fond et elle est génératrice d'usure. Elle devient alors contre-productive; de pitié agissante, elle devient pitié apitoyante. «La sur-réaction empathique permanente, nous rappelle Jeremy Rifkin, peut épuiser l'affectivité, émousser l'empathie et aboutir à un retrait émotionnel de la situation ${ }^{61}$. " Pour éviter ces effets entropiques, Paul Bloom ${ }^{62}$ appelle de ses vœux une compassion rationnelle capable de se soucier de quelqu'un qui souffre sans pour autant soi-même éprouver ce qu'il ressent; une compassion alors très différente de l'empathie qui renvoie à la tendance à se mettre spontanément dans la peau d'autrui, à ressentir ce que ressent l'autre. Et ce pour éviter le traumatisme vicariant $^{63}$ ou le transport de souffrance ${ }^{64}$. "Je crois, pour ma part, dit-il que les meilleurs soignants sont ceux qui se soucient de vous sans ressentir

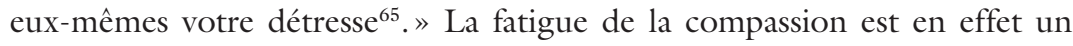
phénomène de plus en plus analysé dans la littérature des sciences sociales ${ }^{66}$. La gestion des émotions en situation professionnelle, dont au premier chef l'exposition à la souffrance et au malheur, prend la forme d'un véritable travail sentimental ${ }^{67}$ supposant l'établissement de règles des sentiments, énoncées par Arlie Horchschild ${ }^{68}$. Ce principe de réglage des sentiments et de la situation se révèle particulièrement pesant pour les agents exposés au public;

60. «On perd de la force quand on compatit», Friedrich Nietsche, L'Antéchrist, Paris, Gallimard, 1983 [1895], p. 22.

61. J. Rifkn, op. cit., p. 121.

62. Tanguy Châtel souligne, pour sa part, en référence notamment à l'accompagnement des mourants, que cet appel entendu de l'autre fragile nous fragilise en retour en raison de notre propre impuissance et insuffisance. Tanguy Châtel, «Éthique du «prendre soin»: sollicitude, care, accompagnement», Emmanuel Hirsch (dir.), Traité de bioéthique, Toulouse, Éditions Érès, 2011.

63. I.e. la souffrance par personne interposée, celle qui est générée par la confrontation soutenue à la souffrance d'un tiers. Pascale Brillon, «Fatigue de la compassion et traumatisme vicariant. Quand la souffrance de nos patients nous bouleverse», Psychologie Québec, vol. 30, n³, 2013, p. 30-35.

64. J. Furtos et C. Laval, op. cit.

65. Paul Bloom «L'empathie, une passion qui tue», Le Temps, 6 février 2017.

66. Cf. notamment A. Gounongbé, op. cit.

67. La notion de travail sentimental a été mise en lumière par Anselm Strauss et ses collègues: «Le travail sentimental est une composante de n'importe quel travail dont l'objet est vivant, sensible, réactif - une composante à la fois nécessaire pour accomplir le travail et fondée sur des considérations humanistes.» Anselm Strauss, Shizuko Fagerhaugh, Barbara Suczek et Carolyn Wiener, «Sentimental Work in the Technological Hospital», Sociology of Health and Illness, vol. 4, n 3, 1982, p. 254. Traduction libre.

68. Arlie Hochshild, «Travail émotionnel, règles de sentiments et structure sociale», Travailler, n 9, 2003, p. 19-49. Angelo Soares, dans le dossier spécial qu'il introduit, parle de «management du travail émotionnel ». Angelo Soares, «Les émotions dans le travail», Travailler, op. cit., p. 9-18. On peut trouver de beaux exemples finement observés de cette gestion des émotions au travail dans deux travaux récents, l'un sur les urgentistes sociaux, l'autre sur les avocats. Daniel Cefaï et Édouard Gardella, L'Urgence sociale en action. Ethnographie du Samu Social 
il suppose tout un répertoire normatif pour faire face aux émotions dans l'exécution des tâches demandées. Et ce, d'autant plus que cette part du travail est souvent méconnue, à tout le moins non reconnue ${ }^{69}$, car difficilement objectivable et faisant l'objet d'une division du travail élastique. La compassion au travail est ainsi vue comme un sentiment moral problématique sur les lieux professionnels quand il y a exposition structurelle à la souffrance d'autrui. D'où toutes ces stratégies professionnelles pour composer en décidant de ne plus la voir, en la tenant à distance ou tout simplement en dosant le degré d'attention à la souffrance d'autrui tant elle peut être un sentiment sans limite se manifestant souvent comme un débordement ${ }^{70}$. On pointe ainsi une usure profonde, douloureuse à la détresse d'autrui, notamment quand, en tant que professionnel ou bénévole, on est trop fréquemment exposé au vécu émotionnel de victimes. La compassion, d'abord ressentie, s'estompe alors pour faire place à des sentiments d'impuissance, d'inutilité de l'intervention, de sensation de ne pas en faire assez, de doute sur le sens de l'action... comme aussi à la nécessité de préserver son intégrité professionnelle et son équilibre personnel.

Cet effet d'effondrement traduit le fait que la compassion empêche l'action en usant la capacité à agir. Pire, même, la souffrance que vit l'autre et que nous recevons et subissons atrophie notre puissance à agir, devant la répétitivité des situations, la vanité de nos efforts, l'incertitude sur nos capacités à pouvoir surmonter l'épreuve. La compassion, en ce sens, amoindrit, paradoxalement, le désir d'agir.

\section{Retour conclusif sur les propriétés sociologiques de la compassion}

Pour conclure, essayons de rassembler les quelques idées directrices qui se dégagent de ce texte dont le projet, même s'il a largement puisé dans la littérature philosophique, était de cerner les propriétés sociologiques de la compassion et de nous éclairer sur ce que la mobilisation de cette notion nous permet de comprendre de l'activité sociale.

Tout d'abord, la compassion, si elle est avant tout une relation, si elle n'est même qu'une relation, est une relation bien particulière. Elle repose sur une inversion de la dissymétrie relationnelle entre l'agent et le patient. Ce faisant, elle rapproche tout en naturalisant la différence. Elle égalise tout

de Paris, Paris, la Découverte, 2011; Janine Barbot et Nicolas Dodier, «Que faire de la compassion au travail? La réflexivité stratégique des avocats à l'audience», Sociologie du travail, vol. 56, nº 3, 2014.

69. Cf. Anselm Strauss et al., op. .cit., mais aussi dans la littérature française Pascale Molinier, «Travail et compassion dans le monde hospitalier », Les Cahiers du genre, $\mathrm{n}^{\circ} 28,2000$, p. 49-70.

70. Cf., à titre d'exemple de ce travail sentimental, le cycle de la distance et de la compassion très tôt identifié par Erving Goffman. Erving Goffman, Asiles. Études sur la condition sociale des malades mentaux et autres reclus, Paris, Les Éditions de Minuit, 1968 [1961]. 
en refusant l'identification. Elle reconnaît autrui comme un sujet autonome tout en n'entendant pas la réciprocité. Elle est une attention forte à un autre vulnérable tout en reposant sur un partage impossible, car seulement imaginable, d'expérience.

Par ailleurs, la dimension spectaculaire de la souffrance, au sens de ce qui attire le regard, est un moteur essentiel de la compassion - Je ne choisis pas de voir, je suis frappé par le malheur d'autrui. Je suis pris, de manière non réfléchie, malgré moi. La compassion provient du fait que nous sommes saisis du dehors, subissant passivement la vue du malheur d'autrui.

Ensuite, la compassion place en son cour la question de l'action, même si elle se caractérise par un rapport négatif à celle-ci. Il ne s'agit ni d'indignation ni d'injustice qui, elles, nous mobilisent. La compassion nous cloue sur place; elle relève de notre impuissance à agir en même temps qu'elle la révèle. C'est de cette incapacité à produire une action transformatrice de la situation de l'autre souffrant que naît la compassion. Elle porte à venir en aide mais n'est pas en soi cette aide car, si elle avait été action, elle ne serait plus puisqu'ayant dépassé le moment de l'impuissance d'agir ${ }^{71}$.

Enfin, la compassion produit des effets tout en n'agissant pas; elle est désir d'action en même temps que fruit de l'incapacité à agir; elle invite à l'engagement mais se dissout dès que celui-ci se concrétise en action; elle est reconnaissance de l'autre par partage médiat de sa souffrance, mais seulement l'instant du spectacle. En donnant à voir une peine subie et, en même temps, en exprimant une impossibilité de rendre, elle est partage. Elle n'engage pas, elle oblige; et en cela, elle est considération car actualisation d'un lien, car manifestation d'une commune appartenance.

D'une certaine manière, en quelque sorte, la compassion est une notion sociologique en trompe-l'œil : elle est avant tout une relation, mais une relation qui ne vise que la relation car l'affliction, quoique (ou parce que) muette et passive, est l'action elle-même. C'est en cela, d'une certaine façon, que, compatir tient lieu de faire, même si, ce faisant, c'est faire beaucoup.

71. C'est pourquoi, il ne faudrait pas confondre, même si l'on se situe dans un champ sémantique voisin, compassion et émotion, car le propre de cette dernière est justement, comme l'étymologie le rappelle, de mettre en mouvement, alors que le propre de la première est de faire sans se mouvoir. 